\title{
TEG-platelet mapping-guided evaluation of anti-phospholipid carrier with unusual clinical presentation and paradoxical laboratory findings
}

\begin{abstract}
Anti-phospholipid syndrome is defined by the combination of clinical thrombosis and detection of persistent anti-phospholipid antibodies (aPL), including lupus anticoagulant (LA). Those who do not meet full clinical criteria of overt thrombosis but still harbor circulating aPL can be characterized as "anti-phospholipid carriers." Regardless of clinical manifestations, aPL can complicate coagulation testing, most commonly by elevating activated partial thromboplastin time (aPTT) and potentially obscuring additional coagulation defects. Platforms for global assays of hemostasis such as thromboelastography (TEG), especially in cases of complex (multiple) coagulopathies, grant a wider scope of detection to help guide more specific probing of individual defects. We report a case of an anti-phospholipid carrier initially evaluated with TEG, revealing paradoxical results suggesting multiple coagulation defects rather than the expected hypercoagulability characteristic of aPL. Directed follow-up testing revealed the patient had an antibody-mediated platelet defect as well as decreased factor XII activity. We present this case as an uncommon manifestation of aPL detected via TEG.
\end{abstract}

Keywords: anti-phospholipid syndrome, anti-phospholipid antibodies, lupus anticoagulant, thromboelastography, platelet mapping, factor XII
Volume 2 Issue I - 2016

Oksana Volod,' Yao Ma,' Sepehr Rokhsar² 'Department of Pathology and Laboratory Medicine, Cedars Sinai Medical Center, USA

${ }^{2}$ Tower Hematology Oncology Medical Group, USA

Correspondence: Oksana Volod, Department of Pathology and Laboratory Medicine, Cedars Sinai Medical Center, Los Angeles, CA, USA, Tel (3I0) 423-547I, Fax (310) 423-0483, Email Oksana.Volod@cshs.org

Received: December 09, 2015 | Published: January 29, 2016
Abbreviations: aCL, anti-cardiolipin antibodies; aB2GPI, anti- $\beta$, glycoprotein-I; aPL, anti-phospholipid antibodies; APS, antiphospholipid syndrome; aPTT, activated partial thromboplastin time; LA, lupus anti-coagulant; TEG, thromboelastography; TEG-PM, thromboelastography with platelet mapping; TIA, transient-ischemic attack; IVF, in-vitro fertilization; PT, prothrombin time

\section{Introduction}

Anti-phospholipid syndrome (APS) is an autoimmune disease and is the most common acquired risk factor for thrombophilia, characterized by venous and/or arterial thrombosis, including pregnancy morbidity. The diagnosis of APS, according to the revised Sapporo criteria, is requisite on the presence of both laboratory detection of anti-phospholipid antibodies (aPL) and clinical features of thrombosis (Table 1). ${ }^{1}$ While the syndrome most often manifests clinically as thrombophilia, rarely aPL can be associated with a bleeding diathesis. ${ }^{2}$ While not necessarily always clinically significant, aPL may also mask other potential underlying coagulopathies by complicating their workup through prolonging aPTT-based coagulation testing. Discriminating between aPL-associated bleeding diathesis and a concurrent coagulopathy can be difficult. Global assays of hemostasis, such as thromboelastography (TEG), are purportedly functional tests of in-vivo coagulation by proxy using whole blood, and can be useful in patients with aPL to discriminate and characterize their effects. We report a case of a patient with strong triple-positive circulating aPL with an unusual clinical presentation not meeting full criteria for APS. Initial use of TEG guided further work-up and more specific characterization of the patient's clinical condition.

Table I Summarized criteria for diagnosis of anti-phospholipid syndrome

APS is present if at least one clinical and one laboratory criteria of the following are met:

Clinical Criteria*

I. Vascular thrombosis, confirmed on imaging or pathology

2. Pregnancy morbidity

a) Unexplained fetal death (>10weeks gestation)

b) Premature birth (<34weeks) due to severe preeclampsia, eclampsia, or placental insufficiency

c) Three or more unexplained miscarriages ( $<10$ weeks gestation)

\section{Laboratory Criteria**}

I. Persistent circulating LA 
Table Continued....

\section{APS is present if at least one clinical and one laboratory criteria of the following are met:}

2. Persistent circulating $\mathrm{aCL} \operatorname{lgG}$ and/or IgM antibodies, in moderate to high titers (>40GPL or MPL)

3. Persistent circulating aB2GPI IgG and/or IgM antibodies, in high titers (>99th percentile)

*Classification of APS should be avoided if less than 12 weeks or more than 5 years separate the positive aPL test and the clinical manifestation.

**Detection of LA, aCL, and/or aB2GPI antibodies must be on two or more occasions, tested at least I 2 weeks apart, measured by lupus anticoagulant sensitive assays and standardized ELISA, respectively.

APS - anti-phospholipid syndrome; LA - lupus anticoagulant; aCL - anticardiolipin antibody; aB2GPI - anti-B2 glycoprotein-I antibody

\section{Case presentation}

39-years-old female with a long standing history of transient ischemia attack (TIA)-like symptoms since age 20 manifesting as ocular migraines with peripheral vision loss and scotoma. Having been evaluated at multiple large institutions, she had been subject to multiple imaging modalities evaluating the cerebral vasculature and parenchyma, showing no evidence of embolic disease, vascular stenosis, abnormal flows or overt cerebral-vascular accidents, although micro-angiopathic changes could not be ruled out. The patient also denies any history of thrombotic events or pregnancy loss, reporting a normal pregnancy conceived by in-vitro fertilization (IVF) and successful delivery. Her labs were remarkable for a persistent lupus anticoagulant (LA) and high titer anti-cardiolipin (>100GPL ref: <20GPL) and anti- $\beta_{2}$ Glycoprotein-I (>100SGU ref: <20SGU) IgG antibodies. PT was normal but aPTT was always elevated $(\sim 60 \mathrm{sec})$. Other thrombophilia workup including: Factor V Leiden gene mutation, Prothrombin II gene mutation, anti-thrombin, Protein $\mathrm{C}$, and Protein S levels were normal. Complete blood counts were normal with low-normal platelet counts $\left(150-20010^{3} / \mu \mathrm{L}\right)$. Despite her high titer anti-phospholipid antibodies, she did not meet full clinical criteria for a diagnosis of APS due to lack of overt thrombotic events. Despite management with aspirin, the patient continued to have TIAlike symptoms.

The patient eventually came to the attention of the hematologist and subsequently coagulation specialist in our institution. We decided to initially evaluate her with a global assay for hemostasis, namely thromboelastography (TEG) and its modification: platelet mapping (TEG-PM). Given the patient's clinical history and positive antiphospholipid workup, we expected an overall hypercoagulable result. Surprisingly, she showed significant combined decreased coagulation factor(s) activity and platelet dysfunction beyond the expected aspirin effect (Figure 1).

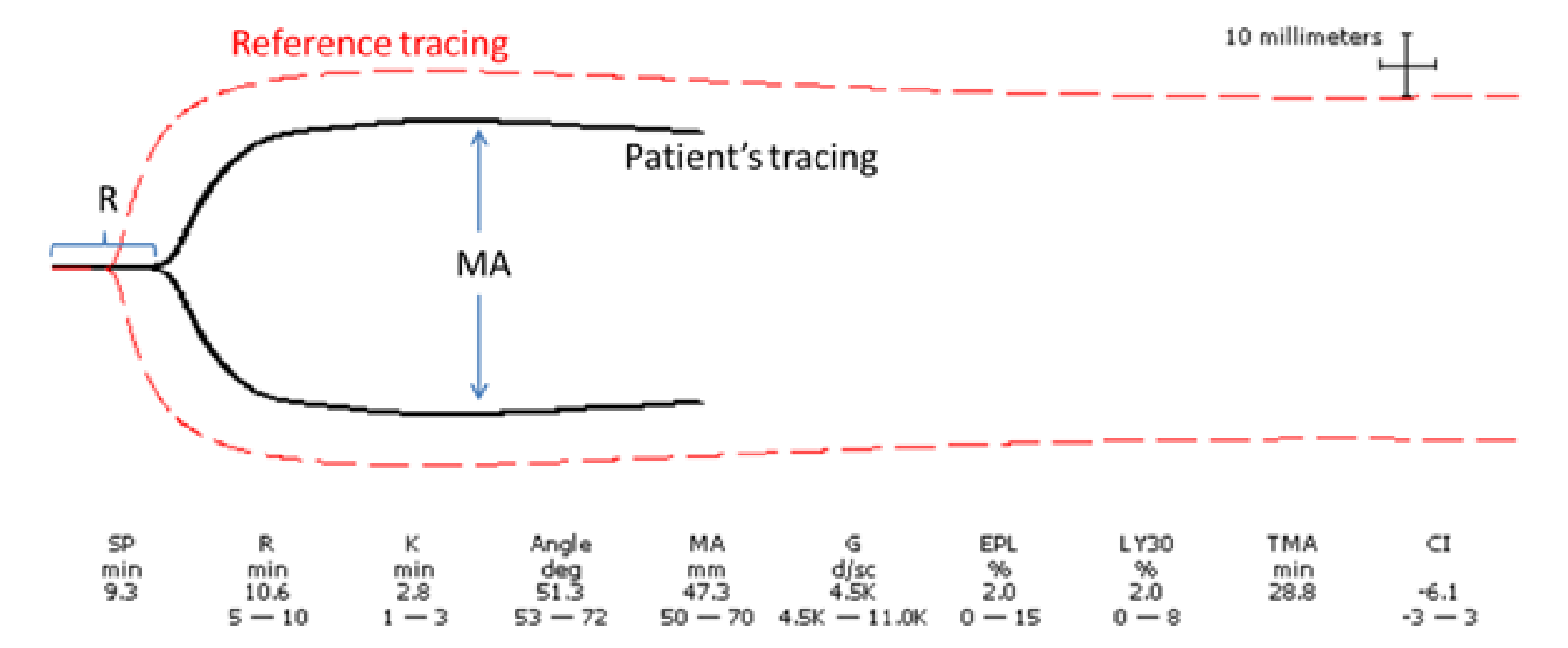

Figure I Basic thromboelastography (TEG) tracing of the patient (solid lines) overlaying a normal reference tracing (dotted lines). The patient's R (reaction) time is prolonged compared to the reference tracing, representing coagulation factor deficiency/inhibition. The MA (maximum amplitude) is also decreased compared to the reference tracing, representing platelet dysfunction. Note that the patient's low-normal platelet count $\left(150 \times 10^{3} / \mu \mathrm{L}\right)$ and aspirin effect alone would not account for this degree of platelet dysfunction, which is corroborated by concurrent testing using TEG with platelet mapping (tracing not pictured here).

To evaluate the platelet component, whole blood (platelet) aggregometry was performed, showing borderline or decreased aggregation and granule secretion globally with all tested agonist agents (Table 2). These findings raised possibility of platelet-reactive antibodies. Further workup included send-out testing for platelet antibody identification panel to a specialized reference laboratory, which yielded a positive screen for platelet-reactive $\operatorname{IgG}$ antibodies or immune complexes, but without specificity for GPIIb/IIIa, GPIb/IX, GPIa/IIa, CD36, or HLA-Class I moieties. Despite these results, the possibility of platelet-specific auto-antibodies could not be excluded using this platform. To evaluate the coagulation factor(s) component, based on her clinical history and laboratory findings (no bleeding, abnormal aPTT, female), factor activity testing was limited to factors XI and XII, which revealed normal factor XI activity (104\%; ref:50$150 \%)$, but decreased factor XII activity (30\%; ref: $50-150 \%)$. In summary, the patient is most accurately characterized at this time as an anti-phospholipid carrier with potentially platelet-reactive and anti-factor XII antibodies. 
Table 2 Patient whole blood (Platelet) aggregometry results

\begin{tabular}{|c|c|c|c|}
\hline Agonist & Patient result (ohm) & Reference range (ohm) & Interpretation* \\
\hline \multicolumn{4}{|c|}{ Platelet Aggregation (Impedance) } \\
\hline Collagen, I $\mu g / m L$ & 18 & $15-27$ & Borderline \\
\hline Collagen, $5 \mu \mathrm{g} / \mathrm{mL}$ & 18 & $|5-3|$ & Borderline \\
\hline $\mathrm{AA}, 0.5 \mathrm{mM}$ & 4 & $5-17$ & Decreased \\
\hline ADP, $10 \mu \mathrm{M}$ & 16 & $6-24$ & Normal \\
\hline Ristocetin, $0.25 \mathrm{mg} / \mathrm{mL}$ & 0 & $>1$ & Normal \\
\hline Ristocetin, $1.0 \mathrm{mg} / \mathrm{mL}$ & 5 & $<5$ & Borderline \\
\hline \multicolumn{4}{|c|}{ Platelet Secretion (ATP release) } \\
\hline Agonist & Patient Result (nmol) & Reference Range (nmol) & Interpretation* \\
\hline Collagen, I $\mu \mathrm{g} / \mathrm{mL}$ & 0.56 & $0.5-1.7$ & Borderline \\
\hline Collagen, $5 \mu \mathrm{g} / \mathrm{mL}$ & 0.59 & $0.9-1.7$ & Decreased \\
\hline $\mathrm{AA}, 0.5 \mathrm{mM}$ & 0.3 & $0.6-1.4$ & Decreased \\
\hline$A D P, 10 \mu M$ & 0.49 & $0.38-1.71$ & Borderline \\
\hline Thrombin, IU & 0.56 & $>0.5$ & Borderline \\
\hline
\end{tabular}

*The patient's platelet aggregometry are resulted here. Platelet aggregometry uses exposure to various agonists to assess platelet reactivity through corresponding receptors. Measurement of impedance is used to assess platelet activation/aggregation, while measuring ATP release assesses platelet secretion function. Globally borderline to decreased results, as seen here, are indicative of a non-specific process blocking agonist binding, possibility indicating antibody interference.

\section{Discussion}

The risk of primary and recurrent thrombosis in APS patients depends on the combination of several factors including:

i. The aPL profile (type(s), level, and persistence of aPL).

ii. Co-existent thrombotic risk factors.

iii. The association with underlying autoimmune diseases, mainly systemic lupus erythematosus. . $^{1,3,4}$

With respect to aPL profile, thrombotic risk increases with triple-positivity of aPL types (LA, anti-cardiolipin, and anti- $\beta_{2}$ Glycoprotein-I), higher titers of aPL, and prolonged persistence of aPL.

Although the laboratory detection of aPL is critical to the diagnosis of APS, their presence alone without definitive clinical symptoms of thrombosis places those asymptomatic patients into the category of "anti-phospholipid carrier" rather than APS. This distinction is important in therapy considerations. A cornerstone of management of APS is long-term antithrombotic therapy (vitamin-K antagonist \pm aspirin $),{ }^{5}$ the duration and intensity of which remains debatable. The reported risk benefits of primary thrombo-prophylaxis in asymptomatic anti-phospholipid carriers are mixed in the literature. ${ }^{4,6,7}$

The difficulty with our particular patient is that although she did not fully meet clinical criteria for overt thrombosis necessary to qualify for the diagnosis of APS, she was still arguably symptomatic given her recurrent TIA-like symptoms, which is known to be associated with APS but is not a diagnostic criterion. This in combination with her high titers and triple-positive aPL places her at high risk for future overt thrombotic events. Such high-risk patients could arguably benefit from thrombo-prophylaxis and appropriate hemostasis testing would help guide this management.

Global assays of hemostasis such as TEG, especially in cases of complex (multiple) coagulopathies, grant a wider scope of detection to help guide more specific probing of individual defects (factor or platelet-driven). Currently, TEG has been used extensively in hemostasis monitoring during major surgeries, trauma, obstetrical complications, and management of deep vein thrombosis; while its modification, TEG with platelet mapping (TEG-PM), is used to monitor anti-platelet activity. Mechanically, TEG measures the changes in visco-elastic properties of whole blood during the process of clot formation and dissolution, providing real-time information about a patient's hemostatic profile in a modality that simulates the invivo environment. ${ }^{8,9}$ The most pertinent parameters of a TEG tracing include reaction time, angle, maximum amplitude, and percent lysis at 30 minutes (Figure 2).
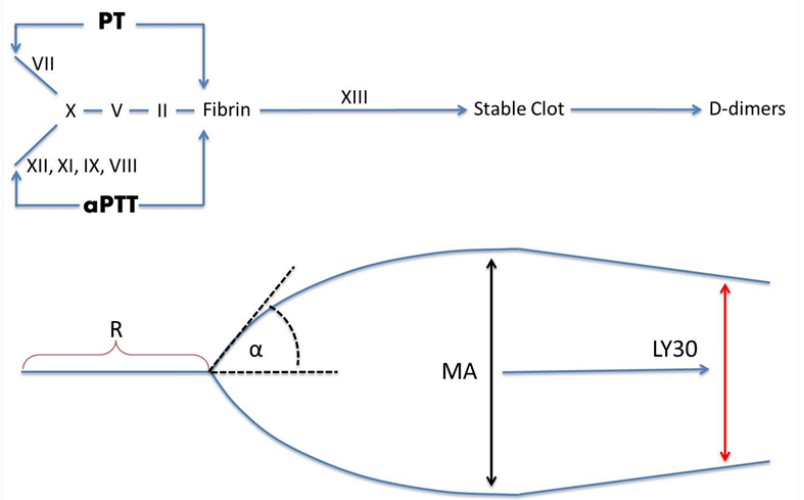

Figure 2 Diagram explaining basic parameters of a thromboelastography tracing and its associated events in clot formation (represented above the tracing).

R (minutes), Reaction time; represents overall coagulation factor activity; prolonged $\mathrm{R}$ represents a factor deficiency.

$\boldsymbol{\alpha}$ (degrees), Angle; represents rate of fibrin formation; decreased angle may represent hypo/dysfibrinogenemia.

MA ( $\mathbf{m m}$ ), Maximum amplitude; represents maximal platelet function and activity; a decreased or increased MA may represent thrombocytopenia/ platelet inhibition or hyperactivity, respectively.

LY30 (\%), Clot lysis at 30min; represents clot stability; decreased LY30 may represent abnormally accelerated fibrinolysis such as in DIC. 
With respect of this patient, given her TIA-like symptoms and strong triple-positive aPL, we expected a hypercoagulable profile on TEG. Instead, TEG and TEG-PM revealed a combined baseline hypocoagulable-state of decreased factor activity and platelet inhibition beyond that which could be attributed to aspirin or her low-normal platelet count $\left(150 \times 10^{3} / \mu \mathrm{L}\right)$. This snapshot guided follow-up testing addressing both the platelet function through platelet aggregometry and selective coagulation factor activity testing. Factors XI and XII were singled out for testing due to the low clinical yield of testing other aPTT-sensitive factors, namely VII and IX, given the patient's lack of bleeding history and female gender (hemophilia A and B are both X-linked recessive disorders). Overall, results were indicative of platelet function inhibition and decreased factor XII activity.

LA and aPL are a heterogenous group that can target multiple different proteins in the coagulation pathway and exhibit various effects that complicate coagulation testing and are usually prothrombotic but rarely associated with a bleeding diathesis. ${ }^{2}$ Specifically, although aPL's interaction with platelets are usually associated with platelet activation and aggregation, ${ }^{10}$ rare instances of LA-mediated inhibition of collagen-induced adhesion and aggregation are reported. ${ }^{11}$ Likewise, LA has also been documented to target factor XII, causing deficiency which is known to prolong aPTT and R values in TEG, but is of unresolved clinical significance. ${ }^{12}$ Primary factor XII deficiency is usually asymptomatic, although it has been reported to have association with arterial and venous thrombosis. Finally, aPL has also been reported to target prothrombin (factor II) leading to accelerated clearance and associated with bleeding. But, in such cases of hypoprothrombinemia, prothrombin time (PT) would be concurrently prolonged as well, which was not the case here.

Taking all these factors in mind together with the results from the various coagulation testing, it can reasonably be assumed that the patient's baseline platelet inhibition can be attributed to plateletreactivity of her aPL, and prolonged $\mathrm{R}$ value is due to factor XII deficiency, although a true deficiency versus inhibition by aPL could not be discriminated with performed testing. Clinically, an interesting speculation can be made that the patient's lack of overt thrombosis, despite the strength and endurance of her triple-positive aPL antibody profile, may be attributed to a sort of tenuous balance between the pro-thrombotic and platelet inhibitory effects of the aPL. Although this balance may be occasionally disrupted, manifesting as TIA-like events, more intense thrombo-prophylaxis is not likely indicated, provided there is no further progression of symptoms. If additional anticoagulation were to be considered, the patient would most benefit from decreasing factor activity (through vitamin-K antagonists or factor inhibitors) rather than through additional platelet inhibition. Limited studies have also shown hydroxychloroquine to have some risk benefit for aPL carriers. ${ }^{7}$ In this case, we demonstrated the utility of TEG in a complex case by framing a global picture of hemostasis that further guided additional workup.

\section{Acknowledgements}

None.

\section{Conflict of interest}

The author declares no conflict of interest.

\section{References}

1. Miyakis $\mathrm{S}$, Lockshin MD, Atsumi $\mathrm{T}$, et al. International consensus statement on an update of the classification criteria for definite antiphospholipid syndrome (APS). J Thromb Haemost. 2006;4(2):295-306.

2. Forastiero R. Bleeding in the anti-phospholipid syndrome. Hematology. 2012;17(Suppl 1):S153-S155.

3. Pengo V, Banzato A, Bison E, et al. What have we learned about antiphospholipid syndrome from patients and anti-phospholipid carrier cohorts? Semin Thromb Hemost. 2012;38(4):322-327.

4. Pengo V, Ruffatti A, Legnani C, et al. Incidence of a first thromboembolic event in asymptomatic carriers of high-risk anti-phospholipid antibody profile: a multicenter prospective study. Blood. 2011;118(17):4714-4718.

5. Les, I G, Ruiz-Irastorza, Khamashta MA. Intensity and duration of anticoagulation therapy in anti-phospholipid syndrome. Semin Thromb Hemost. 2012;38(4):339-347.

6. Erkan D, Harrison MJ, Levy R, et al. Aspirin for primary thrombosis prevention in the anti-phospholipid syndrome: a randomized, doubleblind, placebo-controlled trial in asymptomatic anti-phospholipid antibody-positive individuals. Arthritis Rheum. 2007;56(7):2382-2391.

7. Erkan D, Yazici Y, Peterson MG. et al. Sammaritano L, Lockshin MD (2002) A cross-sectional study of clinical thrombotic risk factors and preventive treatments in antiphospholipid syndrome. Rheumatology (Oxford). 2002;41(8):924-929.

8. Bolliger D, Seeberger MD, Tanaka KA. Principles and practice of thromboelastography in clinical coagulation management and transfusion practice. Transfus Med Rev. 2012;26(1):1-13.

9. Reikvam H. Thrombelastography. Transfus Apher Sci. 2009;40(2):119 123.

10. Willis R, Harris EN, Pierangeli SS. Pathogenesis of the anti-phospholipid syndrome. Semin Thromb Hemost. 2012;38(4):305-321.

11. Ostfeld I, Dadosh-Goffer N, Borokowski S, et al. Lupus anticoagulant antibodies inhibit collagen-induced adhesion and aggregation of human platelets in-vitro. J Clin Immunol. 1992;12(6):415-423.

12. Jones DW, Gallimore MJ, MacKie IJ, et al. Reduced factor XII levels in patients with the anti-phospholipid syndrome are associated with antibodies to factor XII. Br J Haematol. 2000;110(3):721-726. 